\title{
LOWEST EIGENVALUES OF NEARLY CIRCULAR REGIONS*
}

\author{
BY \\ HERBERT B. KELLER AND JOSEPH B. KELLER \\ Institute of Mathematical Sciences, New York University
}

1. Introduction and formulation. Consider a plane, simply connected, closed, domain bounded by the curve

$$
r=R(\theta),
$$

where $R(\theta)=R(\theta+2 \pi)$. It is well known that the problem

$$
\left(\nabla^{2}+\lambda^{2}\right) u(r, \theta)=0, \quad r<R(\theta) ; \quad u(r, \theta)=0, \quad r=R(\theta),
$$

for sufficiently regular curves (1), has a non-trivial solution if and only if $\lambda^{2}$ has one of an infinite sequence of positive real values, the eigenvalues. In many problems of mathematical physics (e.g. waveguides and vibrating membranes) the smallest such value, $\lambda^{2}$, called the principal eigenvalue, is of importance. $\dagger$ In the present paper a method for approximating the principal eigenvalue is considered.

The principal eigenvalue of any domain lies between the principal eigenvalues of the inscribed and circumscribed circles, as is well known. If the principal eigenvalue of the circle of equal area is used instead of that of the circumscribed circle, the same statement applies, as is also well known. The latter statement is sharper in that the equal area circle furnishes a better (i.e., greater) lower bound than the circumscribed circle.

In this paper we attempt to improve the lower bound, that is, to get a still greater lower bound. We do this by introducing a family of functionals of the domain which depend monotonically upon a parameter $\alpha$. For $\alpha=-\infty, 2,+\infty$ this functional yields the principal eigenvalues of the inscribed, equal area, and circumscribed circles respectively. We then conjecture that there exist values of $\alpha$ which furnish better lower bounds than does the equal circle. For almost circular boundaries we find that $\alpha=-1.779 \cdots$ is the best such value.

It seems probable that when $\alpha=-2$, in which case the functional is quite simple, a good approximation which is not necessarily a lower bound, is obtained for $\lambda^{2}$. As an example of the utility of the result, the exact eigenvalues of a rectangle with arbitrary ratio of sides is compared with the approximate expression, given by an integral, for various values of $\alpha$. The agreement is within 3\% for all rectangles when $\alpha=-1$ and even better for nearly square rectangles when $\alpha=-2$.

2. The $\alpha$-mean radius.** The boundary (1) is given parametrically in terms of rectangular coordinates with origin at the pole and $x$-axis on the ray $\theta=0$ by

$$
x(\theta)=R(\theta) \cos \theta, \quad y(\theta)=R(\theta) \sin \theta ; \quad 0 \leq \theta \leq 2 \pi .
$$

*Received July 11, 1953. This work was performed at Washington Square College of Arts and Sciences, New York University, and was supported in part by Contract No. AF-19(122)-1 with the U. S. Air Force through the Air Force Cambridge Research Center, Air Research and Development Command.

$\dagger$ The principal frequency of a membrane or cutoff frequency of TM modes in a waveguide is determined by $\lambda$.

**The analysis of this section holds only for a convex boundary but may be extended to star-shaped domains and perhaps to arbitrary simply connected regions. 
Translating the origin to any point $(h, k)$ within or on the boundary and introducing the new coordinates $x^{\prime}$ and $y^{\prime}$ gives for the new parametric equations of the boundary:

$$
x^{\prime}(\theta)=x(\theta)-h, \quad y^{\prime}(\theta)=y(\theta)-k ; \quad 0 \leq \theta \leq 2 \pi .
$$

In terms of new polar coordinates $(\rho, \phi)$ with $(h, k)$ as pole the boundary is, say,

$$
\rho=P(\phi),
$$

where

$$
x^{\prime}(\phi)=P(\phi) \cos \phi, \quad y^{\prime}(\phi)=P(\phi) \sin \phi ; \quad 0 \leq \phi \leq 2 \pi .
$$

Thus, from (4) and (6),

$$
\phi=\tan ^{-1} \frac{y(\theta)-k}{x(\theta)-h} .
$$

These simple transformations are shown in Fig. 1.

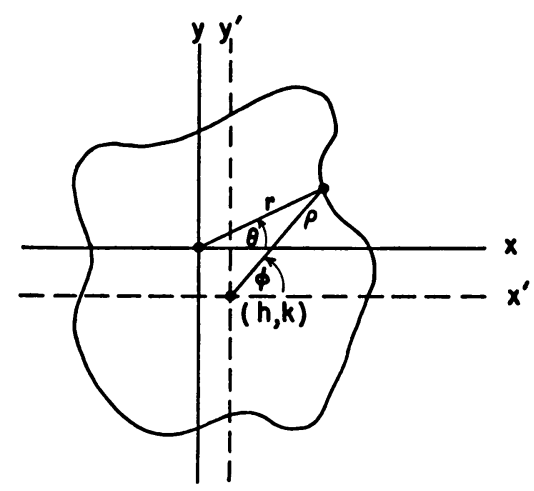

FIg. 1

We now define the " $\alpha$-mean radius with respect to $(h, k)$ " of the boundary curve (1) as

$$
{ }_{h . k}\langle R(\theta)\rangle_{\alpha}=\left\{\frac{1}{2 \pi} \int_{0}^{2 \pi} P^{\alpha}(\phi) d \phi\right\}^{1 / \alpha}
$$

From equations (3)-(7) the above definition becomes

$$
\begin{aligned}
{ }_{h, k}\langle R(\theta)\rangle_{\alpha}= & \left\{\frac{1}{2 \pi} \int_{0}^{2 \pi}\left[R^{2}(\theta)-2 R(\theta)(h \cos \theta+k \sin \theta)+\left(h^{2}+k^{2}\right)\right]^{(\alpha-2) / 2}\right. \\
& \left.\cdot\left[R^{2}(\theta)-R(\theta)(h \cos \theta+k \sin \theta)-\frac{d R}{d \theta}(h \sin \theta-k \cos \theta)\right] d \theta\right\}^{1 / \alpha} .
\end{aligned}
$$

In retaining the limits of integration we have used the periodicity of all functions involved. This expression shows explicitly the dependence of ${ }_{h, k}\langle R(\theta)\rangle_{\alpha}$ on $h, k$ and $R(\theta)$. The " $\alpha$-mean radius", $R_{\alpha}$, for the boundary curve (1) is defined as follows:

$$
\left.\begin{array}{ll}
\alpha \geq 2, & R_{\alpha}=\min _{(h, k) h, k}\langle R(\theta)\rangle_{\alpha} \\
\alpha \leq 2 & R_{\alpha}=\max _{(h, k) h, k}\langle R(\theta)\rangle_{\alpha}
\end{array}\right\} \text { for all }(h, k) \text { within } r=R(\theta) .
$$


The points $(h, k)$ which furnish these extreme values will be called the " $\alpha$-centers" of the cross-section. The case $\alpha=2$ is exceptional since ${ }_{h, k}\langle R(\theta)\rangle_{2}$ is independent of $h$ and $k$; in fact $R_{2}$ is the ordinary mean square radius or the radius of the circle with area equal to that of the given cross-section and any point is a 2-center. For a particular choice of $(h, k)$ it can be shown (see [2], paragraph 6.10) that ${ }_{h, k}\langle R(\theta)\rangle_{\alpha}$ is a monotonic increasing function of $\alpha$ [with slight restrictions (see [2], paragraph 6.6 and 6.10) on the function $R(\theta)$, which are assumed to hold]. By means of the definition (10) it is easily proved that the same must be true of the $\alpha$-mean radius, or explicitly,

$$
R_{\alpha} \leq R_{\beta} \quad \text { for } \quad \alpha<\beta .
$$

The equality holds only if the boundary is a circle. In the appendix it is shown that $R_{\alpha}=a$ for a circle of radius $a$ and the center of the circle is an $\alpha$-center.

Interesting consequences of the definition (10) are obtained if we let $\alpha \rightarrow \pm \infty$. Thus (see [2], paragraph 6.10) from (8)

$$
\left.\begin{array}{l}
{ }_{h, k}\langle R(\theta)\rangle_{\infty}=\max _{\phi} P(\phi) \\
{ }_{h, k}\langle R(\theta)\rangle_{-\infty}=\min _{\phi} P(\phi)
\end{array}\right\} \text { for all } \phi \text { in } 0 \leq \phi \leq 2 \pi .
$$

The corresponding mean radii become, by (10),

$$
\left.\begin{array}{rl}
R_{\infty} & =\min _{(h, k)}\left[\max _{\phi} P(\phi)\right] \\
R_{-\infty} & =\max _{(h, k)}\left[\min _{\phi} P(\phi)\right]
\end{array}\right\} \text { for all }(h, k) \text { within } r=R(\theta) \text { and } \phi \text { in } 0 \leq \phi \leq 2 \pi .
$$

The first expression is the radius of the minimum circle containing the given cross-section (i.e., the circumscribed circle) and the second is the radius of the maximum circle contained within the given cross-section (i.e., the inscribed circle).

3. Bounds for the principal eigenvalue. For a given cross-section (1) consider the family of circles with radii $r=R_{\alpha}$, the $\alpha$-mean radii of the boundary. The principal eigenvalue, $\lambda_{\alpha}$, for any circle is the smallest positive number (see [6], section C) such that $J_{0}\left(\lambda_{\alpha} R_{\alpha}\right)=0$, and thus

$$
\lambda_{\alpha}=j_{01} / R_{\alpha} .
$$

Here $j_{01}$ is the first root of the zero-order Bessel function. From the monotonicity of $R_{\alpha}$, expressed in (11), it is obvious that $\lambda_{\alpha}$ must be a monotonic decreasing function of $\alpha$; that is

$$
\lambda_{\alpha} \geq \lambda_{\beta} \quad \text { for } \quad \alpha<\beta .
$$

It is a well known theorem that for any cross-section the principal eigenvalue is bounded above and below by, respectively, the principal eigenvalues for the inscribed and circumscribed circles. Thus, from (13), this theorem is expressed in our notation as

$$
\lambda_{\infty} \leq \lambda \leq \lambda_{-\infty} .
$$

Here $\lambda$ is taken to be the principal eigenvalue of the arbitrary cross-section (1) and the equalities hold only if this figure is a circle. Further, Faber [4] and Krahn [5] have proved the following theorem: among all cross-sections with the same area, the circle has the least principal eigenvalue. By the previous discussion of the case $\alpha=2$ this theorem becomes in our notation

$$
\lambda_{2} \leq \lambda
$$


the equality again holding only for a circle. From the monotonic property (15) and the theorems expressed in (16) and (17) we have for any cross-section

$$
\lambda_{\alpha} \leq \lambda, \quad \text { for } \quad 2 \leq \alpha \leq \infty .
$$

This may be considered a trivial extension of the theorem of Faber and Krahn to the following

Theorem: Among all cross-sections with the same $\alpha$-mean radius, for any $\alpha$ in $2 \leq \alpha \leq \infty$, the circle has the least principal eigenvalue.

From the monotonic property (15) it is obvious that for a given boundary the closest approximation $\lambda_{\alpha}$, to the true eigenvalue $\lambda$, for the above range of $\alpha$, is $\lambda_{2}$. If the boundary is a circle all $\lambda_{\alpha}$ are equal to the true value, and if not then $\lambda_{2}<\lambda$. Thus we are led, naturally, to seek some $\alpha_{*}<2$ such that for any boundary $\lambda_{\alpha} . \leq \lambda$. This bound would be closer than that obtained from the equal area circle. If such a value could be obtained the above theorem could be extended to the range $\alpha_{*} \leq \alpha \leq \infty$, where $\alpha_{*}<2$, in which case it would be a non-trivial extension of the theorem of Faber and Krahn. We shall proceed to show that for boundaries which are perturbations from a circle such a value does exist and we shall obtain it.

4. Almost-circular boundaries. Let the boundary of a domain be give $n$ by

where

$$
r=R(\theta) \equiv R_{0}+\sum_{j=1}^{\infty} \epsilon^{i} R_{j}(\theta) ;
$$

$$
R_{i}(\theta)=\sum_{n=0}^{\infty}\left[R_{n j} \cos n \theta+S_{n j} \sin n \theta\right], \quad j=1,2, \cdots .
$$

Here $\epsilon$ is a small positive parameter which is a measure of the deviation of the curve from a circle, and $S_{0 j} \equiv 0$. The problem formulated in (2) has been solved for the above boundary, up to second order in $\epsilon$, by various authors (see, [1], Chap. IX, 209-211; [3], Paragraph 6.5(a); [6], section C). The principal eigenvalue is found to be

$$
\begin{aligned}
\lambda=\frac{j_{01}}{R_{0}} & {\left[1-\epsilon\left\{\frac{R_{01}}{R_{0}}\right\}\right.} \\
& \left.+\epsilon^{2}\left\{\left(\frac{R_{01}}{R_{0}}\right)^{2}-\frac{R_{02}}{R_{0}}+\frac{1}{2 R_{0}^{2}} \sum_{n=1}^{\infty}\left[j_{01} \frac{J_{n}^{\prime}\left(j_{01}\right)}{J_{n}\left(j_{01}\right)}+\frac{1}{2}\right]\left(R_{n 1}^{2}+S_{n 1}^{2}\right)\right\}+\epsilon^{3} \cdots\right] .
\end{aligned}
$$

Here $j_{01}$ is defined below (14) and $\left.J_{n}^{\prime}\left(j_{01}\right)=(d / d x) J_{n}(x)\right]_{x=i_{01}}$. In order to compute $\lambda_{\alpha}$, defined by (14), the $\alpha$-mean radius of (19) must first be obtained.

Since the center of a circle is an $\alpha$-center (see appendix) and the boundary (19) is almost circular we assume that its $\alpha$-center is located at some point, $(\epsilon h, \epsilon k)$, near the "center". Then using (19) in (9) and expanding in powers of $\epsilon$ gives

$$
\begin{aligned}
\epsilon h, \epsilon k\langle R(\theta)\rangle_{\alpha}= & R_{0}+\epsilon\left\{0.0\left\langle R_{1}(\theta)\right\rangle_{1}\right\} \\
& +\epsilon^{2}\left\{0.0\left\langle R_{2}(\theta)\right\rangle_{1}+\frac{\alpha-1}{2 R_{0}}\left[_{0.0}\left\langle R_{1}^{2}(\theta)\right\rangle_{1}-{ }_{0.0}\left\langle R_{1}(\theta)\right\rangle_{1}^{2}\right]\right. \\
& +\frac{\alpha-2}{4 R_{0}}\left[h^{2}+k^{2}\right]+\frac{1}{2 \pi \alpha R_{0}}\left[\int_{0}^{2 \pi} \frac{d R_{1}(\theta)}{d \theta}(-h \sin \theta+k \cos \theta) d \theta\right. \\
& \left.\left.-(\alpha-1)^{2} \int_{0}^{2 \pi} R_{1}(\theta)(h \cos \theta+k \sin \theta) d \theta\right]\right\}+\epsilon^{3} \cdots
\end{aligned}
$$


In the first integral above integration by parts gives

$$
\int_{0}^{2 \pi} \frac{d}{d \theta} R_{1}(\theta)(-h \sin \theta+k \cos \theta) d \theta=\int_{0}^{2 \pi} R_{1}(\theta)(h \cos \theta+k \sin \theta) d \theta .
$$

Now the integrals appearing in (22) can be combined to yield the term:

$$
-\frac{(\alpha-2)}{2 \pi R_{0}} \int_{0}^{2 \pi} R_{1}(\theta)(h \cos \theta+k \sin \theta) d \theta .
$$

Let the first two Fourier coefficients of $R_{1}(\theta)$ be denoted by

$$
\begin{aligned}
& R_{11}=\frac{1}{\pi} \int_{0}^{2 \pi} R_{1}(\theta) \cos \theta d \theta, \\
& S_{11}=\frac{1}{\pi} \int_{0}^{2 \pi} R_{1}(\theta) \sin \theta d \theta .
\end{aligned}
$$

Using expressions (23) and (24) all terms, up to second order in $\epsilon$, which involve $h$ and $k$ in (22) become

$$
+\frac{(\alpha-2)}{4 R_{0}}\left\{h^{2}-2 h R_{11}+k^{2}-2 k S_{11}\right\} \text {. }
$$

To find $R_{\alpha}$ we must obtain $h$ and $k$ such that the above expression is a minimum for $\alpha>2$ and a maximum for $\alpha<2$. These values are easily found to be:

$$
\left.\begin{array}{l}
h=R_{11} \\
k=S_{11}
\end{array}\right\} \quad \text { for } \quad \alpha>2 \quad \text { and } \quad \alpha<2 \text {. }
$$

The $\alpha$-mean radius of the boundary (19) is thus given by:

$$
\begin{aligned}
R_{\alpha}=R_{0} & +\epsilon\left\{{ }_{0.0}\left\langle R_{1}(\theta)\right\rangle_{1}\right\}+\epsilon^{2}\left\{{ }_{0.0}\left\langle R_{2}(\theta)\right\rangle_{1}\right. \\
& \left.+\frac{(\alpha-1)}{2 R_{0}}\left[{ }_{0.0}\left\langle R_{1}^{2}(\theta)\right\rangle_{1}-{ }_{0.0}\left\langle R_{1}(\theta)\right\rangle_{1}^{2}\right]-\frac{(\alpha-2)}{4 R_{0}}\left[R_{11}^{2}+S_{11}^{2}\right]\right\}+\epsilon^{3} \ldots .
\end{aligned}
$$

for all $\alpha$, where the $R_{11}$ and $S_{11}$ are given by (24).

The principal eigenvalue of a circle with the above radius is, from (27) in (14):

$$
\begin{aligned}
\lambda_{\alpha}=\frac{j_{01}}{R_{0}} & {\left.\left[1-\epsilon\left\{\frac{0.0}{R_{0}}\right\} R_{1}(\theta)\right\rangle_{1}\right\}+\epsilon^{2}\left\{\left[\frac{0.0}{R_{0}}\right]_{\left.\frac{1}{1}(\theta)\right\rangle_{1}}\right]^{2}-\frac{0.0\left\langle R_{2}(\theta)\right\rangle_{1}}{R_{0}} } \\
& \left.\left.-\frac{(\alpha-1)}{2 R_{0}^{2}}\left[{ }_{0.0}\left\langle R_{1}^{2}(\theta)\right\rangle_{1}-{ }_{0.0}\left\langle R_{1}(\theta)\right\rangle_{1}^{2}\right]+\frac{(\alpha-2)}{4 R_{0}}\left[R_{11}^{2}+S_{11}^{2}\right]\right\}+\epsilon^{3} \ldots\right] .
\end{aligned}
$$

Further if each $R_{i}(\theta)$ is given by a Fourier series as in equation (20) the above principal eigenvalue becomes:

$$
\begin{aligned}
\lambda_{\alpha}=\frac{j_{01}}{R_{0}}\left[1-\epsilon\left\{\frac{R_{01}}{R_{0}}\right\}+\epsilon^{2}\left\{\left(\frac{R_{01}}{R_{0}}\right)^{2}-\frac{R_{02}}{R_{0}}\right.\right. & -\frac{1}{4 R_{0}^{2}}\left(R_{11}^{2}+S_{11}^{2}\right) \\
& \left.\left.\quad-\frac{(\alpha-1)}{4 R_{0}^{2}} \sum_{n=2}^{\infty}\left(R_{n 1}^{2}+S_{n 1}^{2}\right)\right\}+\epsilon^{3} \cdots\right] .
\end{aligned}
$$


To compare this with the true principal eigenvalue for a boundary given by equations (19) and (20) we form the difference $\left(\lambda-\lambda_{\alpha}\right)$ where $\lambda$ is given by (21); this yields

$$
\left(\lambda-\lambda_{\alpha}\right)=\epsilon^{2}\left[\frac{j_{01}}{2 R_{0}^{3}} \sum_{n=2}^{\infty}\left\{j_{01} \frac{J_{n}^{\prime}\left(j_{01}\right)}{J_{n}\left(j_{01}\right)}+\frac{\alpha}{2}\right\}\left(R_{n 1}^{2}+S_{n 1}^{2}\right)\right]+\epsilon^{3} \cdots
$$

Here we have used the fact that

$$
j_{01} \frac{J_{1}^{\prime}\left(j_{01}\right)}{J_{1}\left(j_{01}\right)}=-1
$$

this accounts for the cancellation of the terms involving $R_{11}$ and $S_{11}$. In order that $\lambda_{\alpha}$ be a lower bound on the true eigenvalue the difference given by (30) must be positive. Also the best such lower bound must be that for which $\alpha=\alpha_{0}$, the least $\alpha$ for which this difference is still non-negative (since $\lambda_{\alpha}$ is a monotonic decreasing function of $\alpha$ ).

To make (30) positive for all $R_{n 1}$ and $S_{n 1}$ all coefficients of $\left(R_{n 1}^{2}+S_{n 1}^{2}\right)$ in (30) must be non-negative; or

Then $\alpha_{0}$ is given by:

$$
j_{01} \frac{J_{n}^{\prime}\left(j_{01}\right)}{J_{n}\left(j_{01}\right)} \geq-\frac{\alpha}{2} ; \quad \text { for } \quad n=2,3, \cdots .
$$

$$
\alpha_{0}=-2 \underset{(n=2,3,4, \cdots)}{\text { g.l.b. }}\left[j_{01} \frac{J_{n}^{\prime}\left(j_{01}\right)}{J_{n}\left(j_{01}\right)}\right] .
$$

To find the greatest lower bound in the above expression we use*

$$
x \frac{J_{n}^{\prime}(x)}{J_{n}(x)}=n-\sum_{\nu=1}^{\infty} \frac{2 x^{2}}{j_{n \nu}^{2}-x^{2}},
$$

where $j_{n}$ is the $\nu$-th zero of the $n$-th order Bessel function. Thus we must consider

$$
j_{01} \frac{J_{n}^{\prime}\left(j_{01}\right)}{J_{n}\left(j_{01}\right)}=n-\sum_{\nu=1}^{\infty} \frac{2 j_{01}^{2}}{j_{n v}^{2}-j_{01}^{2}} ; \quad n=2,3,4, \cdots
$$

However, (see [7], p. 508 (2)) for fixed $\nu$ the $j_{n \nu}$ are monotonic increasing functions of $n$ and $j_{n \nu}>j_{01}$ for $n \geq 2, \nu \geq 1$. Thus as $n$ increases each term of the sum in (35), and hence the sum, decreases. Therefore the least value of (35) for $n \geq 2$ occurs for $n=2$. Using this value (33) gives

$$
\alpha_{0}=-2 j_{01} \frac{J_{2}^{\prime}\left(j_{01}\right)}{J_{2}\left(j_{01}\right)}=\left(4-j_{01}^{2}\right)=-1.779 \cdots
$$

We have thus proved the following result: For any curve in the neighborhood of a circle the principal eigenvalue is greater than or equal to that of a circle with the same $\alpha_{0}$ mean radius, up to second order in $\epsilon$. This lower bound is the best possible in the sense that for any $\alpha<\alpha_{0}$ there exist curves for which the inequality does not hold. Furthermore this result yields a lower bound which is closer to the true eigenvalue than that given by a circle of equal area. These results are expressed in our notation as follows:

$$
\lambda_{2}<\lambda_{\alpha_{\bullet}} \leq \lambda, \quad \lambda_{\alpha_{\bullet}}=\frac{j_{n_{1}}}{R_{\alpha_{0}}}
$$

*See [7] p. 498 (1). The argument of this paragraph is essentially that of [3], paragraph $6.5(\mathrm{~b})$. For a different proof see [6!; section F. 
If an approximation to the principal eigenvalue is desired, rather than a lower bound, a value of $\alpha$ less than $\alpha_{0}$ should be employed since then the terms in the "error" given by (30) will not all be positive, and may cancel. The.value $\alpha=-2$ seems reasonable, for too small a value would yield a negative error. Furthermore, the calculation of the $\alpha$-mean radius is relatively simple for $\alpha=-2$.

We further conclude that if there exists an $\alpha .<2$ which furnishes a lower bound for all cross-sections, not only those near a circle, it must lie in the range

$$
\alpha_{0}=\left(4-j_{01}^{2}\right) \leq \alpha_{*}<2 .
$$

If not, the above example shows that we could assign Fourier coefficients (and hence determine a boundary) in (30) such that $\lambda_{\alpha} .>\lambda$.

5. Application to rectangles. The eigenvalues for $\downarrow$ rectangular cross-section are: known exactly and thus provide a test of the approximations just obtained.

Let the rectangle be located with its axes of symmetry coincident with the coordinate: axis and its sides of length 2 and $2 \sigma$ respectively. Then the boundary may be expressed in polar form as $r=R(\theta)$ with:

$$
\begin{array}{lr}
R(\theta)=\frac{1}{\cos \theta}, & -\beta \leq \theta \leq \beta, \\
R(\theta)=\frac{\sigma}{\sin \theta}, & \beta \leq \theta \leq \pi-\beta, \\
R(\theta)=\frac{-1}{\cos \theta}, & \pi-\beta \leq \theta \leq \pi+\beta, \\
R(\theta)=\frac{-\sigma}{\sin \theta}, & \pi+\beta \leq \theta \leq 2 \pi-\beta,
\end{array}
$$

where $\beta=\tan ^{-1} \sigma$.

The $\alpha$-mean radius becomes, from (9), (10) and (37):

$$
R_{\alpha}=\left\{\frac{2}{\pi}\left[\int_{0}^{\beta} \frac{1}{\cos ^{\alpha} \theta} d \theta+\sigma^{\alpha} \int_{\beta}^{\pi / 2} \frac{1}{\sin ^{\alpha} \theta} d \theta\right]\right\}^{1 / \alpha}
$$

Here we have used the assumption that the $\alpha$-center lies on a line of symmetry if such exists. The $\alpha$-mean radii are, for $\alpha=2,1,-1$, and -2 :

$$
\begin{aligned}
R_{2} & =\left(\frac{4 \sigma}{\pi}\right)^{1 / 2}, \\
R_{1}= & \frac{2}{\pi}\left[\ln \left(1+\sigma^{2}\right)^{1 / 2}-\sigma \ln \frac{\left(1+\sigma^{2}\right)^{1 / 2}-1}{\left(1+\sigma^{2}\right)^{1 / 2}+1}\right], \\
R_{-1} & =\frac{\pi}{2} \frac{\sigma}{\left(1+\sigma^{2}\right)^{1 / 2}}, \\
R_{-2} & =(\pi)^{1 / 2} \sigma\left[\sigma+\beta \sigma^{2}+\left(\frac{\pi}{2}-\beta\right)\right]^{-1 / 2} .
\end{aligned}
$$

From these we may compute the principal eigenvalues of the circles with the corresponding radii (by means of (14)). The exact principal eigenvalue for the rectangle is (see [3], P. 253)

$$
\lambda=\frac{\pi}{2} \frac{\left(1+\sigma^{2}\right)^{1 / 2}}{\sigma} .
$$


We thus obtain the following ratios:

$$
\begin{aligned}
\frac{\lambda}{\lambda_{2}} & =\frac{(\pi)^{1 / 2}}{j_{01}}\left(\sigma+\frac{1}{\sigma}\right)^{1 / 2}, \\
\frac{\lambda}{\lambda_{1}} & =\frac{1}{j_{01}}\left(1+\frac{1}{\sigma^{2}}\right)^{1 / 2}\left[\ln \left(1+\sigma^{2}\right)^{1 / 2}-\sigma \ln \frac{\left(1+\sigma^{2}\right)^{1 / 2}-1}{\left(1+\sigma^{2}\right)^{1 / 2}+1}\right], \\
\frac{\lambda}{\lambda_{-1}} & =\frac{\pi^{2}}{4 j_{01}} \\
\frac{\lambda}{\lambda_{-2}} & =\frac{\pi^{3 / 2}}{2 j_{01}}\left(1+\sigma^{2}\right)^{1 / 2}\left[\sigma+\beta \sigma^{2}+(\pi / 2-\beta)\right]^{-1 / 2} ; \beta \equiv \tan ^{-1} \sigma .
\end{aligned}
$$

These ratios give a measure of the closeness of the approximation by various circles as a function of the ratio of the sides of the rectangle (see Fig. 2). It is interesting to note that the $(-1)$-mean radius has a constant ratio of approximation for all rectangles. This is all the more remarkable since Polya and Szegö (see [3], paragraph 5.9(b)) have obtained, for a rectangle, an upper bound on the principal frequency (eigenvalue) which also has a constant ratio of approximation. This ratio is

$$
\frac{\lambda^{*}}{\lambda}=\left(\frac{\lambda}{\lambda_{-1}}\right)^{1 / 2}\left(\frac{2}{j_{01}}\right)^{1 / 2}=\frac{\pi}{j_{01}(2)^{1 / 2}},
$$

where $\lambda^{*}$ is the upper bound on the principal frequency. This value is exactly that of the asymptote to. the $\lambda / \lambda_{-2}$ curve of Fig. 2 .

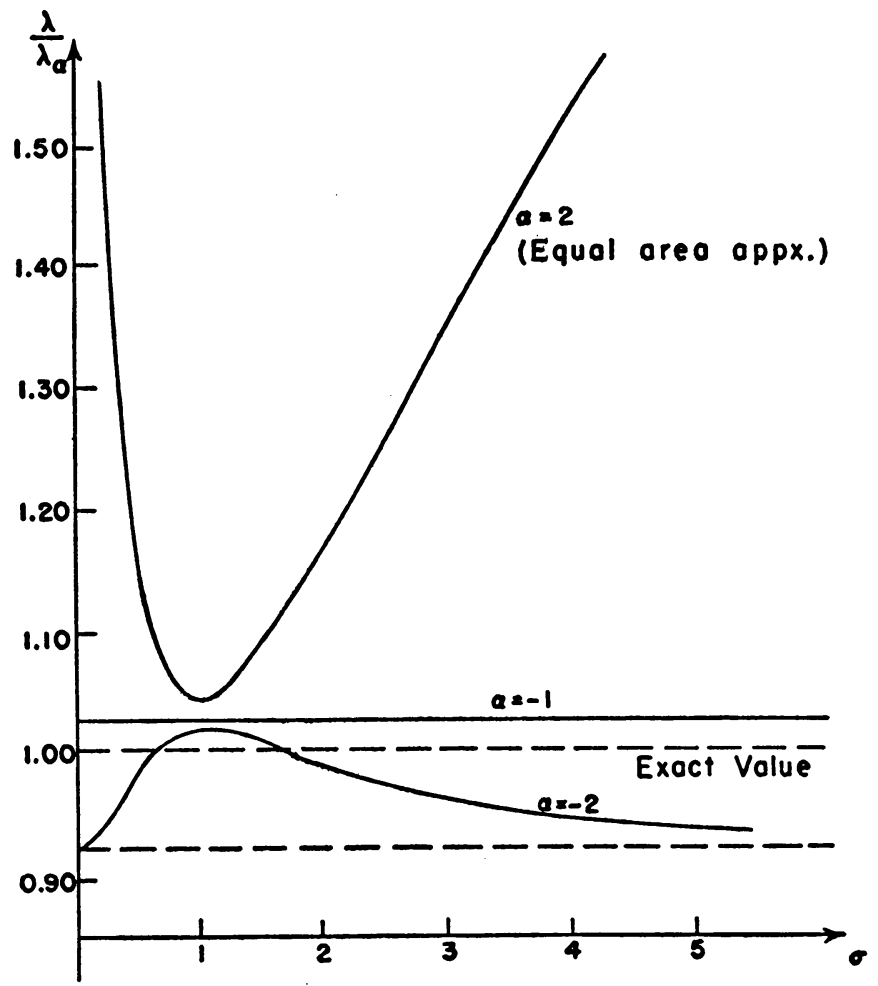

FIG. 2. Ratio of Approximation 


\section{Appendix}

Mean radius of a circle. In finding the $\alpha$-mean radius of an almost circular boundary it has been assumed that the $\alpha$-center is in the neighborhood of the center of the unperturbed circle. This assumption is valid if it can be shown that for a circular boundary the center is an $\alpha$-center. It is easily shown that the center furnishes a relative maximum $(\alpha<2)$ or a minimum ( $\alpha>2)$ but from the definition given by equation (10) we require an absolute extreme. It is shown below that the center of a circle is an $\alpha$-center for all $\alpha$.

By symmetry we may place the center on the $x$-axis when the boundary is a circle given by $R(\theta)=a$ (see Fig. 3). This yields for the " $\alpha$-mean radius with respect to $h$ "

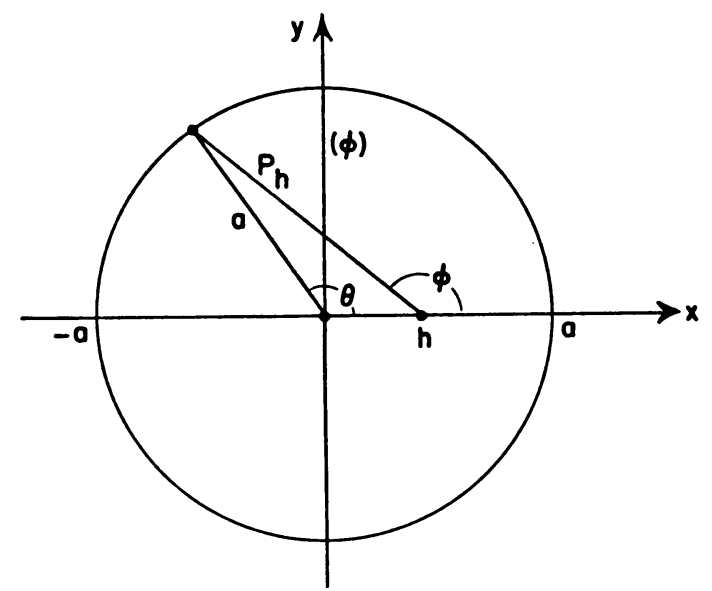

FIG. 3

$$
\begin{aligned}
{ }_{h}\langle a\rangle_{\alpha} & =\left\{\frac{1}{2 \pi} \int_{0}^{2 \pi} P_{h}^{\alpha}(\phi) d \phi\right\}^{1 / \alpha} \\
& =\left\{\frac{1}{2 \pi} \int_{0}^{2 \pi}\left[a^{2}-2 a h \cos \theta+h^{2}\right]^{\alpha-2 / 2}\left[a^{2}-a h \cos \theta\right] d \theta\right\}^{1 / \alpha},
\end{aligned}
$$

where $-a \leq h \leq a$. If we let $\alpha \rightarrow \pm \infty$, which may be done for all $h$ since $P_{h}(\phi)$ vanishes at one point at most (see [2], paragraph 6.6 and 6.10), there results:

$$
\begin{gathered}
{ }_{h}\langle a\rangle_{\infty}=\underset{\phi}{\operatorname{maximum}} P_{h}(\phi)=a+|h|, \\
{ }_{h}\langle a\rangle_{-\infty}=\underset{\phi}{\operatorname{minimum}} P_{h}(\phi)=a-|h| .
\end{gathered}
$$

Furthermore we have by the remarks under equations (10):

$$
{ }_{h}\langle a\rangle_{2}=a \text {. }
$$

Using equations (A.2) and (A.3) and the fact that ${ }_{h}\langle a\rangle_{\alpha}$ is a monotonic increasing function of $\alpha$ we must have:

$$
\left.\begin{array}{l}
a \leq{ }_{h}\langle a\rangle_{\alpha} \leq a+|h|, \quad \alpha>2 \\
a-|h| \leq{ }_{h}\langle a\rangle_{\alpha} \leq a, \quad \alpha<2
\end{array}\right\}-a \leq h \leq a .
$$


The $\alpha$-mean radius defined by (10) is now from (A.4),

$$
\begin{array}{ll}
R_{\alpha}=\min _{h h}\langle a\rangle_{\alpha}=a ; & a>2, \\
R_{\alpha}=\max _{h h}\langle a\rangle_{\alpha}=a ; & a<2 .
\end{array}
$$

This value is attained in either case for $h=0$; i.e., the center of the circle.

\section{REFERENCES}

1. Rayleigh, Theory of sound, Vol. I, Dover, 1945.

2. G. H. Hardy, J. E. Littlewood and G. Polya, Inequalities, Camb. Univ. Press, 1934.

3. G. Polya, and G. Szegö, Isoperimetric inequalities in mathematical physics, Princeton U. Press, 1951.

4. G. Faber, Beweis dass unter allen homogenen Membranen von gleicher Fläche und gleicher Spannung die kreisförmige den tiefsten Grundton gibt, Sitzungsberiche, Bayr. Ak. Wiss, 1923, 169-172.

5. E. Krahn, Über eine von Rayleigh formulierte Minimaleigenschaft des Kreises, Math. 94, 97-100 (1924).

6. H. B. Keller and J. B. Keller, Eigenvalues of nearly circular wave-guides, Res. Rpt. TW-17, NYU, Math. Res. Grp., Wash. Sq. College of Arts and Sci., 1952.

7. G. N. Watson, A treatise on the theory of Bessel functions (Second edition), Camb. Univ. Press, 1948. 